STUDI

FRANCESI

\section{Studi Francesi}

Rivista quadrimestrale fondata da Franco Simone

175 (LIX | I) | 2015

Varia

Aa. Vv., Le Réalisme et ses paradoxes (1850-1900). Mélanges offerts à Jean-Louis Cabanès, sous la direction de Gabrielle Chamarat et Pierre-Jean Dufief

\title{
Maria Emanuela Raffi
}

\section{OpenEdition}

Journals

Edizione digitale

URL: http://journals.openedition.org/studifrancesi/551

DOI: 10.4000/studifrancesi.551

ISSN: 2421-5856

Editore

Rosenberg \& Sellier

Edizione cartacea

Data di pubblicazione: 1 aprile 2015

Paginazione: 177-180

ISSN: 0039-2944

Notizia bibliografica digitale

Maria Emanuela Raffi, «Aa. VV., Le Réalisme et ses paradoxes (1850-1900). Mélanges offerts à Jean-Louis Cabanès, sous la direction de Gabrielle Chamarat et Pierre-Jean Dufief », Studi Francesi [Online], 175 (LIX |I) | 2015, online dal 01 avril 2015, consultato il 18 septembre 2020. URL : http:// journals.openedition.org/studifrancesi/551 ; DOI : https://doi.org/10.4000/studifrancesi.551

Questo documento è stato generato automaticamente il 18 settembre 2020.

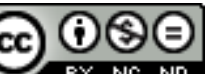

Studi Francesi è distribuita con Licenza Creative Commons Attribuzione - Non commerciale - Non opere derivate 4.0 Internazionale. 


\title{
Aa. Vv., Le Réalisme et ses paradoxes (1850-1900). Mélanges offerts à Jean- Louis Cabanès, sous la direction de Gabrielle Chamarat et Pierre-Jean Dufief
}

\author{
Maria Emanuela Raffi
}

\section{NOTIZIA}

Aa. Vv., Le Réalisme et ses paradoxes (1850-1900). Mélanges offerts à Jean-Louis Cabanès, sous la direction de Gabrielle CHAMARAT et Pierre-Jean DUfIEF, Paris, Classiques Garnier, 2014, pp. 510.

1 Dopo la ricca introduzione dei due curatori, che ripercorre l'ampia opera critica dell'illustre specialista dell'Ottocento, Jean-Louis Cabanès, cui questi Mélanges sono dedicati, trentatré contributi raggruppati in quattro parti distinte, costituiscono il corposo insieme del volume, di cui si renderà conto, di necessità, un po' rapidamente.

2 «Écoles, savoir et institutions», prima parte, è inaugurata da Bernard vouilloux con un saggio consacrato soprattutto a Le Négatif di Cabanès e alle considerazioni di ordine generale che vi sono contenute, da cui prende spunto per una serie di osservazioni sulla dyschronie che caratterizza i rapporti fra letteratura e storia da una parte, fra critica e letteratura dall'altra (Les styles collectifs dans l'histoire et devant l'historiographie).

In La question du réalisme entre 1848 et 1855, Gabrielle CHAMARAT entra nel vivo della «querelle» sul realismo, considerando prima la corrispondenza fra George Sand e Champfleury, divisi nella valutazione dell'arte di Courbet, poi il volume Le Réalisme, dello stesso Champfleury, messo a confronto con Les Nuits d'octobre di Nerval. Ne 
emerge l'idea nervaliana di «réalisme» come «contemplation distraite», «composition d'observation attentive et de rêverie» sorretta dalla soggettività di «un acte de parole».

4 La nozione cabanesiana di «hétérographie» è al centro del contributo di Antonia FONYI, Mérimée hétérographe, in cui è fortemente sottolineata la doppia natura dell'opera di Mérimée, divisa nettamente fra fiction e ispirazione storica e, all'interno di questa partizione, tentata con eguale energia dal principio del piacere («la sauvagerie») e dal principio di realtà («la civilisation»).

5 L'âme, l'atome et le Moi. Physique et métaphysique dans "Les Misérables" di Pierre LAFORGUE parte dall'affermazione hugoliana che «la misère est matière» e ne esamina tutte le implicazioni, particolarmente evidenti nello scritto del 1860 Philosophie. Commencement d'un livre, arrivando fino ad affermare la natura materiale dell'anima stessa, che Hugo associa costantemente all'atomo, particella di base della materia. A questa associazione corrisponde tuttavia anche un altro parallelismo, quello fra anima e «moi», che per Laforgue sottrae l'anima ad una totale materializzazione e la confronta, nella necessità della definizione personale, ad un infinito indubbiamente di natura spirituale.

Come essere riconosciuto come autore quando per tutti si è soprattutto il discepolo di Flaubert? È la domanda che si pone Laure HeLms nel suo studio su Maupassant («Soyons des originaux». Maupassant et l'héritage flaubertien), in cui, dopo aver mostrato i profondi legami delle famiglie dei due scrittori e la formazione letteraria costruita da Maupassant sull'insegnamento costante di Flaubert, analizza anche il processo di «déflaubertisation» che segue la morte del maestro e che culmina in Le Horla. In confronto all' «homme de Lettres» Flaubert, Maupassant «est d'abord et avant tout celui qui s'efface $[. .$.$] une pure ouverture sur le monde».$

7 In Et si Huysmans avait récrit Champfleury? Sur les sources éventuelles de "Sac au dos" et d"'À vau l'eau", Jean-Marie SEILLAN si interroga, sulla base di numerose somiglianze sia tematiche che di scelta enunciativa, sulla possibile filiazione delle due novelle di Huysmans da due racconti di Champlfleury: Histoire du lieutenant Valentin contenuta nel volume Madame Eugénio del 1874 e Le Cabaret de ma tante Péronne del 1867.

Dominique PÉTY, che rinvia ai suoi precedenti studi sul tema degli “interni”, propone qui (Du règne des reliques au retour du vivant) un rapido percorso - da Baudelaire a Saint-John Perse, passando per Verlaine, Mallarmé, Zola e Verhaeren - in quel «repliement dans l'intérieur privé» che caratterizza tutta una parte della letteratura degli ultimi decenni del secolo e tende a dissolversi solo agli inizi del novecento.

9 Ad un romanzo di Georges Darien ("Bas les cœurs", ou l'histoire inconvenante) è dedicato il contributo di Béatrice LAVILLE, che mette in luce il particolare tipo di realismo realizzato nell'opera, consacrata alla guerra del 1870 e alla Commune senza che questi avvenimenti storici straordinari compaiano mai nel testo, sostituiti dalle loro modeste e spesso indegne ricadute nel quotidiano.

10 Cénacles et Barricades di vincent LAISNEY prende in esame l'alleanza, celebrata da opere artistiche e letterarie, ma mai verificata effettivamente nelle rivolte fra il 1827 e il 1871, fra l'artista e l'operaio in rivolta. Non è nella folla - afferma Laisney - ma nei Cénacles che vanno cercati gli scrittori dell'epoca, dove si forma progressivamente un'élite artiste, gruppo di scrittori isolati nel doppio involucro del Cenacolo e della propria scrittura e per nulla propensi a mescolarsi alle lotte politiche.

11 Gilles BONNET inaugura la seconda parte del volume («Fantaisies post-romantiques») collegandosi all'interesse di Cabanès per $i$ «fantaisistes» per soffermarsi sulla figura di 
Pierrot sceptique, pièce scritta da Huysmans in collaborazione con Léon Hénnique nel 1881, di cui indica le origini nella descrizione di una serata alle Folies Bergère del 1879 inserita da Huysmans in Croquis parisiens (Duel aux Folies Bergère).

12 Le pantomime di Champfleury sono l'oggetto dello studio di Michela Lo FEUDo ( «Égyptiens» aux Funambules) che si interroga su un progetto dell'autore per una Histoire des beaux-arts égyptiens, mai realizzata, in cui vede tuttavia un profondo legame con l'arte della pantomima attraverso l'interesse di Champfleury - e dell'epoca - per i geroglifici. "Tout comme l'art égyptien, la pantomime se donne à lire comme un spectacle muet nourri d'idées», un codice da decifrare.

sandrine BERTHELOT presenta un romanzo, precursore del poliziesco, di Charles Barbara ( "L'Assassinat du Pont-Rouge" de Charles Barbara), autore poco noto «ami de Baudelaire, lecteur de Poe et d'Hoffmann», esponente della bohème parigina, che era stato fra le letture di Jean-Louis Cabanès.

La Fantaisie, qualità rimessa in onore dagli studi di Cabanès, è analizzata da AnneSimone DUfIEF nei racconti di Daudet come strumento utilizzato dallo scrittore per elaborare episodi tratti dal folklore provenzale, dando vita ad un genere ibrido, espressione di una "sensibilité littéraire qui s'est épanouie entre l'ironie romantique et la fantaisie décadente» (Folklore et fantaisies dans les contes de Daudet).

Yves REBOUL (Rimbaud fantaisiste? À propos de "Juillet") riprende l'idea di fantaisie applicandola all'opera di Rimbaud e in particolare ad una poesia che titola con decisone «Juillet», nonostante le incertezze che accompagnano tale scelta anche secondo l'edizione Guyaux del 2009. Il carattere solo superficialmente «fantaisiste» del testo poetico, il cui manoscritto è stato recuperato e studiato da Murphy, è rivelato da Reboul attraverso i diversi livelli di un'analisi testuale densa e precisa, che svela l'articolazione progressiva di un «discours rien moins que ludique» e di un più nascosto progetto di rivolta, là dove non si è visto spesso, semplicisticamente, che un testo illeggibile. Corbière "poète insomniaque» e approfondisce i singolari legami fra scrittura poetica $\mathrm{e}$ insonnia. I due testi poetici di Corbière su questo tema vanno al di là di ciò che altri poeti (Hugo, Belmontet, «Le Cygne» di Baudelaire e altri) hanno proposto in precedenza: la sua insonnia è «une dormeveille enfin euphorique», creativa.

17 Un po' esterno all'ambito francese che caratterizza il volume, il contributo di Vérane PARTENSKY, Carlo Gozzi ou la disparition de l'écrivain, riprende il tema della «fantaisie» in relazione alle fiabe teatrali dello scrittore italiano e alla loro presenza nella letteratura francese dopo la pubblicazione nel 1865 del Théâtre fiabesque de Carlo Gozzi.

Lo studio di Bertrand MARQUER Vers un 'fantastique réel' media il passaggio all'ampia parte del volume dedicata ai Goncourt, prendendo in esame l'aspetto fantastico del romanzo La Faustin del superstite Edmond, che l'A. definisce «fantastique dans l'étude du vrai», caratterizzato da un'ottica clinica molto diffusa nei romanzi di fine secolo.

19 La terza parte, dedicata ai Goncourt, autori ampiamente studiati da Jean-Louis Cabanès - «Les Goncourt ou l'écriture dédoublée» - inizia con "Germinie Lacerteux" des Goncourt, in cui sophie MÉNARD analizza l'organizzazione del tempo nel romanzo attorno all'implacabile avvenimento della morte della figlia. «Les temps du social, du rituel et du quotidien» scandiscono per l'A. l'organizzazione «proleptique» del racconto.

silvia DISEGNI prende in esame, in Le choc du 'double' registre, la lingua utilizzata dai Goncourt, oggetto di polemiche e censure, con un particolare approfondimento del caso 
di Renée Mauperin. È soprattutto la duplicità, «la pratique langagière complexe et mixte», spesso attribuita a personaggi femminili, ad essere rimproverata ai due autori, poiché essa tende a superare e vanificare «la repartition en classes et [les] lieux communs correspondants».

21 Ancora su Germinie Lacerteux, lo studio di Alain vaILLANT (Jules et Edmond de Goncourt, frères de charité), dato per acquisito il carattere «polyphonique» del romanzo realista del xIX secolo, indaga le soluzioni formali che i romanzieri hanno scelto per unificare le diverse voci in un insieme testuale sufficientemente omogeneo. Collocati fra l'amalgama personaggio/narratore prodotto da Flaubert con il discorso indiretto libero e la confusione di piani dello "style direct libre» di Zola, i Goncourt elaborano con il loro "style artiste» la trascrizione di una voce popolare incarnata nella fisicità dell'autore, anche se a distanza e attraverso un soggetto strutturalmente sdoppiato.

Éléonore REVERZY (Les "Germinie Lacerteux") propone una ricognizione nella ricezione di Germinie Lacerteux fra il 1865 e il 1886, raccogliendo un «dossier de presse» di ventisei articoli legati prima alle reazioni de «l'école moderne» che condanna la bruttezza e l'immoralità presenti nel romanzo, poi, vent'anni più tardi, alla battaglia naturalista che l'opera sostiene, sottolineando i documenti che sono alla base della fiction e il suo valore di «biographie d'histoire moderne».

Al conte Armand de Pontmartin, autore dei Jeudis de Madame Charbonneau e critico letterario in possesso di «une verve maligne», qui accostata a quella dell'opera Hommes de lettres (poi Charles Demailly) dei Goncourt, è dedicato l'articolo di Jean-Didier WAGNEUR Armand de Pontmartin, un grand homme de province en province.

La Fille Élisa è l'oggetto dello studio successivo (Élisa, histoire d'une fille di Mireille DoTTINORSINI ); il romanzo "scandaloso" di Edmond de Goncourt è visto come l'iniziatore del genere «romans de filles», racconti su prostitute, del cui silenzio sociale e sentimentale l'autore si fa portavoce, pur nella singolarità della protagonista prostituta e omicida, sola nel suo percorso «de bordel en bordel, d'atelier en atelier carcéral».

«Pourquoi le monde moderne est-il triste?». La domanda attraversa lo scritto di stéphanie CHAMPEAU - L'artiste et la mélancolie - e soprattutto il Journal dei Goncourt, che rispondono tracciando il deprimente quadro della gioventù borghese contemporanea preda di un'idea di progresso che la responsabilizza e la intristisce in un continuo calcolo sul futuro. In questo contesto, l'artista è per l'A. colui che riesce ad uscire dalla concentrazione su di sé «pour cet au-delà de soi, qui est soi et n'est pas soi».

Pierre-Jean DUFIEF concentra la sua attenzione sulle ultime opere di Edmond de Goncourt, raccolte di articoli, di lettere del fratello, di prefazioni teoriche (Les Goncourt et les écritures programmatiques), approfondendo particolarmente il rapporto fra la scrittura delle lettere e quella delle «préfaces». Pur mantenendo le affinità di genere evidenti (la «lettre» per Jules et la «préface» per Edmond), Dufief sottolinea fortemente l'interazione dei due generi nella scrittura dei Goncourt: "pratique constante du recyclage, du couper-coller, de la reformulation».

«Le motif de la nourriture» è presente in molte scene dei romanzi dei Goncourt, consentendo a Joëlle PONNIER di esplorare i documenti utilizzati, l'innato buon gusto degli autori, le loro abitudini personali nonché «la technique du contrepoint chére à l'écriture réaliste/naturaliste» di ancorare gli elementi più drammatici del racconto ad una scena volutamente banale come un pasto (La table dans les romans des Goncourt). 

letteratura e arti visive, prima in generale, definendo le due visioni antitetiche di sorellanza fra le forme espressive (ut pictura poesis) o di competizione nel rapporto con la realtà oggettiva («paragone»), poi nell'opera dei Goncourt L'art du dix-huitième siècle. Ciò che preme all'A. è il reperimento del «Tipping Point» dei Goncourt, cioè il momento in cui la visione strutturante del principio ut pictura poesis dei due autori, relativa al procedimento della creazione, lascia il posto all'inevitabile confronto dei prodotti artistici. di Henri MITTERAND, Genèse des "Rougon-Macquart". Le lieu des différences. Partendo dai suoi passati tentativi di trovare una classificazione cronologica dei dossiers préparatoires del grande insieme narrativo zoliano, lo studioso propone un'esplorazione orizzontale dei sedici dossiers, che rivela tre motivi portanti: la «quête de la différence», sorta di sfida a Balzac, a Flaubert, ai Goncourt; il motivo dell'ereditarietà, connesso all'idea di «famille»; infine, il motivo «arboriforme» per cui la discendenza famigliare si trasforma in «arbre bifide» portatore di una continua virtuale conflittualità.

colette BECKER (Le langage silencieux des manuscrits zoliens) cerca nei dossiers préparatoires zoliani soprattutto la comunicazione muta dei segni grafici: variazioni di scrittura che rivelano lo spostamento di parti da un dossier all'altro e la stratificazione dei capitoli; «hésitations et difficultés» nella scrittura, fogli incollati, cancellature, sottolineature di varie epoche, correzioni con penne e matite; infine appunti e annotazioni a margine, tutti segni di una presenza viva e costante dell'autore.

31 In Le vice et le vitriol, la riflessione di Paolo TORTONESE parte dalla citazione di Taine in epigrafe alla seconda edizione di Thérèse Raquin. Il legame fra morale e materia, che l'epigrafe, sparita nelle edizioni successive, implicava («le vice et la vertu sont des produits, comme le vitriol et le sucre»), viene sostituito da Zola, nella préface del romanzo, da quello fra caractère («moral et dramatique») e tempérament («physique puis psychologique»), con una forte predilezione, nella scelta letteraria, per il secondo. Il rapporto dialettico fra Zola e Taine è attentamente collocato dall'A. nell'ambito dell'ampio dibattito del tempo fra spiritualisti e materialisti.

motivo delle «rovine» guida il percorso di Véronique CNOCKAERT nell'opera di Zola e la porta rapidamente a scoprire che il vero interesse dello scrittore è per le «ruines vivantes», esemplificate qui in due figure emblematiche: Tante Dide e Irma d'Anglars (Ruines de chair). Forse il carattere più interessante di questi personaggi fisicamente devastati è la loro capacità, come scrive l'A., «de faire signe vers une antériorité qui n'est plus mais qui insiste et persiste», di estendersi nel tempo, come segno di radicamento e di rinnovamento, come le rovine di pietra.

33 Stéphane GOUGeLmanN approfondisce in Le naturaliste, l'homosexuel et «l'éloquence de la vérité» un problema affrontato da Zola con disagio e molte resistenze ideologiche («un inverti est un désorganisateur de la famille, de la nation, de l'humanité»), accettato probabilmente solo per amore della verità e per rispetto della sincera passione espressa dall'autore del Roman d'un inverti-né, di cui Zola fa pubblicare il manoscritto con una sua prefazione fondata su una lettera ricevuta da un altro scrittore omosessuale.

"Fécondité", conte, légende, féerie chiude il volume dedicato a Cabanès con uno studio di Jacques Noiray che, da specialista di Zola e dell'ultimo Zola, rileva l'«expansion de l'immagination et de la narration» che caratterizza l'opera dello scrittore a partire da 
Paris e classifica Fécondité come «légende» a causa delle sue volute «invraisemblances et simplifications» nonché del suo schematismo antitetico. Anche la féerie, genere teatrale molto amato nel xIX secolo, è chiamata in causa con le sue convenzioni esibite, la sua imprecisa collocazione temporale, lo statuto simbolico dei personaggi, cui corrisponde uno stile semplice e quasi ingenuo, prodotto da un romanziere divenuto ormai «libre créateur de fictions». 